\title{
Further results of the estimate of growth of entire solutions of some classes of algebraic differential equations
}

\author{
Qi Jianming ${ }^{1,3}$, Li Yezhou ${ }^{2}$ and Yuan Wenjun ${ }^{3^{*}}$
}

\footnotetext{
* Correspondence: gzywj@tom.com ${ }^{3}$ School of Mathematics and Information Science, Guangzhou University, Guangzhou 510006, People's Republic of China Full list of author information is available at the end of the article
}

\begin{abstract}
In this article, by means of the normal family theory we estimate the growth order of entire solutions of some algebraic differential equations and improve the related results of Bergweiler, Barsegian, and others. We also estimate the growth order of entire solutions of a type system of a special algebraic differential equations. We give some examples to show that our results are sharp in special cases.

Mathematica Subject Classification (2000): Primary 34A20; Secondary 30D35.

Keywords: Meromorphic functions, Nevanlinna theory, Normal family, Growth order, Algebraic differential equation
\end{abstract}

\section{Introduction and main results}

Let $f(z)$ be a meromorphic function in the complex plane. We use the standard notation of the Nevanlinna theory of meromorphic functions and denotes the order of $f(z)$ by $\lambda(f)$ (see [1-3]).

Let $\mathbb{C}$ be the whole complex domain. Let $D$ be a domain in $\mathbb{C}$ and $\mathcal{F}$ be a family of meromorphic functions defined in $D . \mathcal{F}$ is said to be normal in $D$, in the sense of Montel, if each sequence $\left\{f_{n}\right\} \subset \mathcal{F}$ has a subsequence $\left\{f_{n_{j}}\right\}$ which converse spherically locally uniformly in $D$, to a meromorphic function or $\infty$ (see [1]).

In general, it is not easy to have an estimate on the growth of an entire or meromorphic solution of a nonlinear algebraic differential equation of the form

$$
P\left(z, w, w^{\prime}, \ldots, w^{(k)}\right)=0,
$$

where $P$ is a polynomial in each of its variables.

A general result was obtained by Gol'dberg [4]. He obtained

Theorem 1.1. All meromorphic solutions of algebraic differential equation (1.1) have finite order of growth, when $k=1$.

For a half century Bank and Kaufman [5] and Barsegian [6] gave some extensions or different proofs, but the results have not changed. Barsegian [7] and Bergweiler [8] have extended Gol'dberg's result to certain algebraic differential equations of higher order. In 2009, Yuan et al. [9], improved their results and gave a general estimate of order of $w(z)$, which depends on the degrees of coefficients of differential polynomial for $w(z)$. In order to state these results, we must introduce some notations: $m \in \mathbb{N}=$

\section{Springer}

(c) 2012 Jianming et al; licensee Springer. This is an Open Access article distributed under the terms of the Creative Commons Attribution License (http://creativecommons.org/licenses/by/2.0), which permits unrestricted use, distribution, and reproduction in any medium, provided the original work is properly cited. 
$\{1,2,3, \ldots\}, r_{j} \in \mathbb{N}_{0}=\mathbb{N} \cup\{0\}$ for $j=1,2, \ldots, m$, and put $r=\left(r_{1}, r_{2}, \ldots, r_{m}\right)$. Define $M_{r}[w]$ (z) by

$$
M_{r}[w](z):=\left[w^{\prime}(z)\right]^{r_{1}}\left[w^{\prime \prime}(z)\right]^{r_{2}} \cdots\left[w^{(m)}(z)\right]^{r_{m}},
$$

with the convention that $M_{\{0\}}[w]=1$. We call $p(r):=r_{1}+2 r_{2}+\ldots+m r_{m}$ the weight of $M_{r}[w]$. A differential polynomial $P[w]$ is an expression of the form

$$
P[w](z):=\sum_{r \in I} a_{r}(z, w(z)) M_{r}[w]
$$

where the $a_{r}$ are rational in two variables and $I$ is a finite index set. The weight $\operatorname{deg} P$ $[w]$ of $P[w]$ is given by $\operatorname{deg} P[w]:=\max _{r \in l} p(r) \cdot \operatorname{deg}_{z, \infty} a_{r}$ denotes the degree at infinity in variable $z$ concerning $a_{r}(z, w) . \operatorname{deg}_{z, \infty} a:=\max _{r \in l} \max \left\{\operatorname{deg}_{z, \infty} a_{r}, 0\right\}$.

Theorem 1.2. [9] Let $w(z)$ be a meromorphic function in the complex plane, $n \in \mathbb{N}, P$ $[w]$ be a polynomial with the form (1.2) $n>\operatorname{deg} P[w]$. If $w(z)$ satisfies the differential equation $\left[w^{\prime}(z)\right]^{n}=P[w]$, then the growth order $\lambda:=\lambda(w)$ of $w(z)$ satisfies

$$
\lambda \leq 2+\frac{2 \operatorname{deg}_{z, \infty} a}{n-\operatorname{deg} P[w]}
$$

Recently Qi et al. [10] further improved Theorem 1.2 as below.

Theorem 1.3. Let $w(z)$ be a meromorphic function in the complex plane and all zeros of $w(z)$ have multiplicity at least $k(k \in \mathbb{N}), P[w]$ be a polynomial with the form (1.2) and $n k q>\operatorname{deg} P[w](n \in \mathbb{N})$. If $w(z)$ satisfies the differential equation $\left[Q\left(w^{(k)}(z)\right)\right]^{n}=P$ $[w]$, then the growth order $\lambda:=\lambda(w)$ of $w(z)$ satisfies

$$
\lambda \leq 2+\frac{2 \operatorname{deg}_{z, \infty} a}{n q k-\operatorname{deg} P[w]},
$$

where $Q(z)$ is a polynomial with degree $q$.

In this article, we first give a small upper bound for entire solutions.

Theorem 1.4. Let $w(z)$ be an entire function in the complex plane and all zeros of $w$ (z) have multiplicity at least $k(k \in \mathbb{N}), P[w]$ be a polynomial with the form (1.2) and $n k q>\operatorname{deg} P[w](n \in \mathbb{N})$. If $w(z)$ satisfies the differential equation $\left[Q\left(w^{(k)}(z)\right)\right]^{n}=P[w]$, then the growth order $\lambda:=\lambda(w)$ of $w(z)$ satisfies

$$
\lambda \leq 1+\frac{\operatorname{deg}_{z, \infty} a}{n q k-\operatorname{deg} P[w]},
$$

where $Q(z)$ is a polynomial with degree $q$.

Example 1 For $n=2$, entire function $w(z)=e^{z^{2}}$ satisfies the following algebraic differential equation

$$
\left(w^{\prime \prime}\right)^{2}=4 w^{2}+16 z^{2} w^{2}+8 z^{3} w^{\prime} w
$$

we know $\operatorname{deg}_{z, \infty} a=3, \operatorname{deg} P[w]=2$, So $\lambda=2 \leq 1+\frac{3}{2 \times 2-1}=2$. This example illustrates that Theorem 1.4 is an extending result of Theorem 1.3 and our result is sharp in the special cases.

By Theorem 1.4, we immediately have the following corollaries. 
Corollary 1.5. Let $w(z)$ be an entire function in the complex plane and all zeros of $w$ $(z)$ have multiplicity at least $k(k \in \mathbb{N}), P[w]$ be a differential polynomial with constant coefficients in variable $w$ or $\operatorname{deg}_{z, \infty} a_{t} \leq 0(t \in I)$ in the (1.2) and $n k q>\operatorname{deg} P[w](n \in$ $\mathbb{N})$. If $w(z)$ satisfies the differential equation $\left[Q\left(w^{(k)}(z)\right)\right]^{n}=P[w]$, then the growth order $\lambda:=\lambda(w)$ of $w(z)$ satisfies $\lambda \leq 1$, where $Q(z)$ is a polynomial with degree $q$.

Corollary 1.6. Let $w(z)$ be an entire function in the complex plane and all zeros of $w$ (z) have multiplicity at least $k(k \in \mathbb{N}), P[w]$ be a polynomial with the form (1.2) and $n k>\operatorname{deg} P[w](n \in \mathbb{N})$. If $w(z)$ satisfies the differential equation $[H(w(z))]^{n}=P[w]$, then the growth order $\lambda:=\lambda(w)$ of $w(z)$ satisfies

$$
\lambda \leq 1+\frac{\operatorname{deg}_{z, \infty} a}{n k-\operatorname{deg} P[w]},
$$

where $H(w(z))=w^{(k)}(z)+b_{k-1} w^{(k-1)}(z)+b_{k-2} w^{(k-2)}(z)+\ldots+b_{1} w(z)+b_{0}$ and $b_{k-1}, \ldots, b_{0}$ are constants.

In 2009, Gu et al. [11] investigated the growth order of solutions of a type systems of algebraic differential equations of the form

$$
\left\{\begin{array}{l}
\left(w^{\prime} 2\right)^{m_{1}}=a(z) w_{1}^{(n)} \\
\left(w_{1}^{(n)}\right)^{m_{2}}=P\left[w_{2}\right]
\end{array}\right.
$$

where $m_{1}, m_{2}$ are the non-negative integer, $a(z)$ is a polynomial, $P\left[w_{2}\right]$ is defined by (1.2).

They obtained the following result.

Theorem 1.7. Let $w=\left(w_{1}, w_{2}\right)$ be the meromorphic solution vector of a type systems of algebraic differential equations of the form (1.3), if $m_{1} m_{2}>\operatorname{deg} P\left(w_{2}\right)$, then the growth orders $\lambda\left(w_{i}\right)$ of $w_{i}(z)$ for $i=1,2$ satisfy

$$
\lambda\left(w_{1}\right)=\lambda\left(w_{2}\right) \leq 2+\frac{2\left(v+\operatorname{deg}_{z, \infty} a\right)}{m_{1} m_{2}-\operatorname{deg} P\left(w_{2}\right)}
$$

where $v=\operatorname{deg}(a(z))^{m_{2}}$.

Qi et al. [10] also consider the similar result to Theorem 1.7 for the systems of the algebraic differential equations

$$
\begin{cases}\left(Q\left(w_{2}^{(k)}(z)\right)\right)^{m_{1}} & =a(z) w_{1}^{(n)} \\ \left(w_{1}^{(n)}\right)^{m_{2}} & =P\left(w_{2}\right),\end{cases}
$$

where $Q(z)$ is a polynomial with degree $q$.

They obtained the following result.

Theorem 1.8. Let $w=\left(w_{1}, w_{2}\right)$ be a meromorphic solution of a type systems of algebraic differential equations of the form (1.4), if $m_{1} m_{2} q k>\operatorname{deg} P\left(w_{2}\right)$, and all zeros of $w_{2}$ (z) have multiplicity at least $k(k \in \mathbb{N})$, then the growth orders $\lambda\left(w_{i}\right)$ of $w_{i}(z)$ for $i=1,2$ satisfy

$$
\lambda\left(w_{1}\right)=\lambda\left(w_{2}\right) \leq 2+\frac{2\left(v+\operatorname{deg}_{z, \infty} a\right)}{m_{1} m_{2} q k-\operatorname{deg} P\left(w_{2}\right)},
$$

where $v=\operatorname{deg}(a(z))^{m_{2}}$.

Similarly we have a small upper bounded estimate for entire solutions below. 
Theorem 1.9. Let $w=\left(w_{1}, w_{2}\right)$ be an entire solution of a type systems of algebraic differential equations of the form (1.4), if $m_{1} m_{2} q k>\operatorname{deg} P\left(w_{2}\right)$, and all zeros of $w_{2}(z)$ have multiplicity at least $k(k \in \mathbb{N})$, then the growth orders $\lambda\left(w_{i}\right)$ of $w_{i}(z)$ for $i=1,2$ satisfy

$$
\lambda\left(w_{1}\right)=\lambda\left(w_{2}\right) \leq 1+\frac{v+\operatorname{deg}_{z, \infty} a}{m_{1} m_{2} q k-\operatorname{deg} P\left(w_{2}\right)},
$$

where $v=\operatorname{deg}(a(z))^{m_{2}}$.

By Theorem 1.9, we immediately obtain a corollary below.

Corollary 1.10. Let $w=\left(w_{1}, w_{2}\right)$ be an entire solution of a type systems of algebraic differential equations of the form

$$
\left\{\begin{array}{l}
\left(H\left(w_{2}\right)\right)^{m_{1}}=a(z) w_{1}^{(n)} \\
\left(w_{1}^{(n)}\right)^{m_{2}}=p\left(w_{2}\right),
\end{array}\right.
$$

where $H(w(z))=w^{(k)}(z)+b_{k-1} w^{(k-1)}(z)+b_{k-2} w^{(k-2)}(z)+\ldots+b_{0}$ and $b_{k-1}, \ldots, b_{0}$ are constants. If $m_{1} m_{2} q k>\operatorname{deg} P\left(w_{2}\right)$, and all zeros of $w_{2}(z)$ have multiplicity at least $k(k \in \mathbb{N})$, then the growth orders $\lambda\left(w_{i}\right)$ of $w_{i}(z)$ for $i=1,2$ satisfy

$$
\lambda\left(w_{1}\right)=\lambda\left(w_{2}\right) \leq 1+\frac{v+\operatorname{deg}_{z, \infty} a}{m_{1} m_{2} q k-\operatorname{deg} P\left(W_{2}\right)},
$$

where $v=\operatorname{deg}(a(z))^{m_{2}}$.

Example 2 Set $w_{1}(z)=e^{z}+c, w_{2}(z)=e^{z}$ satisfy a type systems of algebraic differential equations of the form

$$
\left\{\begin{array}{l}
\left(w_{2}^{(k)}\right)=w_{1}^{(n)} \\
\left(w_{1}^{(n)}\right)^{5}=\left(w_{2}\right)^{3}\left(w_{2}^{\prime}\right)^{2}
\end{array}\right.
$$

where $c$ is a constant, $m_{1}=1, m_{2}=5, v=0, \operatorname{deg}_{z, \infty} a=0$, and $\operatorname{deg} P\left(w_{2}\right)=2$. The (1.6) satisfies the $m_{1} m_{2}=5>2=\operatorname{deg} P\left(w_{2}\right)$. So $\lambda\left(w_{1}\right)=\lambda\left(w_{2}\right)=1 \leq 1$. So the conclusion of Theorem 1.9, Corollary 1.10 may occur and our results are sharp in the special cases.

\section{Preliminary lemmas}

In order to prove our result, we need the following lemmas. The first one extends a famous result by Zalcman [12] concerning normal families. Zalcman's lemma is a very important tool in the study of normal families. It has also undergone various extensions and improvements. The following is one up-to-date local version, which is due to Pang and Zaclman [13].

Lemma $2.1[13,14]$ Let $\mathcal{F}$ be a family of meromorphic (analytic) functions in the unit disc $\Delta$ with the property that for each $f \in \mathcal{F}$, all zeros of multiplicity at least $k$. Suppose that there exists a number $A \geq 1$ such that $\left|f^{(k)}(z)\right| \leq A$ whenever $f \in \mathcal{F}$ and $f$ $=0$. If $\mathcal{F}$ is not normal in $\Delta$, then for $0 \leq \alpha \leq k$, there exist

1. a number $r \in(0,1)$;

2. a sequence of complex numbers $z_{n},\left|z_{n}\right|<r$; 
3. a sequence of functions $f_{n} \in \mathcal{F}$;

4. a sequence of positive numbers $\rho_{n} \rightarrow 0^{+}$;

such that $g_{n}(\xi)=\rho_{n}^{-\alpha} f_{n}\left(z_{n}+\rho_{n} \xi\right)$ converges locally uniformly (with respect to the spherical metric) to a non-constant meromorphic (entire) function $g(\xi)$ on $\mathbb{C}$, and moreover, the zeros of $g(\xi)$ are of multiplicity at least $k, g^{\#}(\xi) \leq g^{\sharp}(0)=k A+1$. In particular, $g$ has order at most 2 . In particular, we may choose $w_{n}$ and $\rho_{n}$, such that

$$
\rho_{n} \leq \frac{2}{\left[f_{n}^{\#}\left(w_{n}\right)\right]^{\frac{1}{1+|\alpha|}}}, \quad f_{n}^{\#}\left(w_{n}\right) \geq f_{n}^{\#}(0) .
$$

Here, as usual, $g^{\#}(\xi)=\frac{\left|g^{\prime}(\xi)\right|}{1+|g(\xi)|^{2}}$ is the spherical derivative. For $0 \leq \alpha<k$, the hypothesis on $f^{(k)}(z)$ can be dropped, and $k A+1$ can be replaced by an arbitrary positive constant.

Lemma 2.2 [15] Let $f(z)$ be holomorphic in whole complex plane with growth order $\lambda:=\lambda(f)>1$, then for each $0<\mu<\lambda-1$, there exists a sequence $a_{n} \rightarrow \infty$, such that

$$
\lim _{n \rightarrow \infty} \frac{f^{\#}\left(a_{n}\right)}{\left|a_{n}\right|^{\mu}}=+\infty
$$

\section{Proof of the results}

Proof of Theorem 1.4 Suppose that the conclusion of theorem is not true, then there exists an entire solution $w(z)$ satisfies the equation $[Q(w(z))]^{n}=P[w]$. such that

$$
\lambda>1+\frac{\operatorname{deg}_{z, \infty} a}{n q k-\operatorname{deg} P[w]} .
$$

By Lemma 2.2 we know that for each $0<\rho<\lambda-1$, there exists a sequence of points $a_{m} \rightarrow \infty(m \rightarrow \infty)$, such that $(2.1)$ is right. This implies that the family $\left\{w_{m}(z):=w\left(a_{m}\right.\right.$ $+z)\}_{m \in \mathbb{N}}$ is not normal at $z=0$. By Lemma 2.1, there exist sequences $\left\{b_{m}\right\}$ and $\left\{\rho_{m}\right\}$ such that

$$
\left|a_{m}-b_{m}\right|<1, \quad \rho_{m} \rightarrow 0,
$$

and $g_{m}(\zeta):=w_{m}\left(b_{m}-a_{m}+\rho_{m} \zeta\right)=w\left(b_{m}+\rho_{m} \zeta\right)$ converges locally uniformly to a nonconstant entire function $g(\zeta)$, which order is at most 2, all zeros of $g(\zeta)$ have multiplicity at least $k$. In particular, we may choose $b_{m}$ and $\rho_{m}$, such that

$$
\rho_{m} \leq \frac{2}{w^{\#}\left(b_{m}\right)}, \quad w^{\sharp}\left(b_{m}\right) \geq w^{\sharp}\left(a_{m}\right) .
$$

According to (2.1) and (3.1)-(3.3), we can get the following conclusion:

For any fixed constant $0 \leq \rho<\lambda-1$, we have

$$
\lim _{m \rightarrow \infty} b_{m}^{\rho} \rho_{m}=0 .
$$

In the differential equation $\left[Q\left(w^{(k)}(z)\right)\right]^{n}=P[w(z)]$, we now replace $z$ by $b_{m}+\rho_{m} \zeta$. Assuming that $P[w]$ has the form (1.2). Then we obtain

$$
\left(Q\left(w^{(k)}\left(b_{m}+\rho_{m} \zeta\right)\right)\right)^{n}=\sum_{r \in I} a_{r}\left(b_{m}+\rho_{m} \zeta, g_{m}(\zeta)\right) \rho_{m}^{-p(r)} M_{r}\left[g_{m}\right](\zeta)
$$


where

$$
\begin{aligned}
Q\left(w^{(k)}\left(b_{m}+\rho_{m} \zeta\right)\right)=\rho_{m}^{-q k}[ & \left(g_{m}^{(k)}\right)^{q}(\zeta)+\rho_{m}^{k} a_{q-1}\left(g_{m}^{(k)}\right)^{q-1}(\zeta)+ \\
& \left.+\cdots+\rho_{m}^{(q-1) k} a_{1} g_{m}^{(k)}(\zeta)+\rho_{m}^{q k} a_{0}\right] .
\end{aligned}
$$

Hence we deduce that

$$
\begin{array}{r}
\rho_{m}^{-n q k}\left[\left(g_{m}^{(k)}\right)^{q}(\zeta)+\rho_{m}^{k} a_{q-1}\left(g_{m}^{(k)}\right)^{q-1}(\zeta)+\cdots+\rho_{m}^{q k} a_{0}\right]^{n} \\
=\sum_{r \in I} a_{r}\left(b_{m}+\rho_{m} \zeta, g_{m}(\zeta)\right) \rho_{m}^{-p(r)} M_{r}\left[g_{m}\right](\zeta) .
\end{array}
$$

Therefore

$$
\begin{aligned}
{\left[\left(g_{m}^{(k)}\right)^{q}(\zeta)\right.} & \left.+\rho_{m}^{k} a_{q-1}\left(g_{m}^{(k)}\right)^{q-1}(\zeta)+\cdots+\rho_{m}^{q k} a_{0}\right]^{n} \\
& =\sum_{r \in I} \frac{a_{r}\left(b_{m}+p_{m} \zeta, g_{m}(\zeta)\right)}{b_{m}^{\operatorname{deg}_{z, \infty} a_{r}}}\left[b_{m}^{\frac{\operatorname{deg}_{z, \infty} a_{r}}{n q k-p(r)}} \rho_{m}\right]^{n q k-p(r)} M_{r}\left[g_{m}\right](\zeta)
\end{aligned}
$$

Because $0 \leq \rho=\frac{\operatorname{deg}_{z_{\infty, \infty}} a_{r}}{n q-p(r)} \leq \frac{\operatorname{deg}_{z_{z, \infty}} a}{n q k-\operatorname{deg} P[w]}<\lambda-1, p(r)<n q k$, for every fixed $\zeta \in \mathbb{C}$, if $\zeta$ is not the zero of $g(\zeta)$, by (3.4) then we can get $g^{(k)}(\zeta)=0$ from (3.5). By the all zeros of $g(\zeta)$ have multiplicity at least $k$, this is a contradiction.

The proof of Theorem 1.4 is complete.

Proof of Theorem 1.9 By the first equation of the systems of algebraic differential equations (1.4), we know

$$
w_{1}^{(n)}=\frac{\left(Q\left(w_{2}^{(k)}(z)\right)\right)^{m_{1}}}{a(z)} .
$$

Therefore we have

$$
\lambda\left(w_{1}\right)=\lambda\left(w_{2}\right) .
$$

If $w_{2}$ is a rational function, then $w_{1}$ must be a rational function, so that the conclusion of Theorem 2 is right. If $w_{2}$ is a transcendental meromorphic function, by the systems of algebraic differential equations (1.3), then we have

$$
\left(Q\left(w_{2}^{(k)}\right)\right)^{m_{1} m_{2}}=(a(z))^{m_{2}} P\left(w_{2}\right) .
$$

Suppose that the conclusion of Theorem 2 is not true, then there exists an entire vector $w(z)=\left(w_{1}(z), w_{2}(z)\right)$ which satisfies the system of equations (1.4) such that

$$
\lambda:=\lambda\left(w_{2}\right)>1+\frac{v+\operatorname{deg}_{z, \infty} a}{m_{1} m_{2} q k-\operatorname{deg} P\left(w_{2}\right)},
$$

By Lemma 2.2 we know that for each $0<\rho<\lambda-1$, there exists a sequence of points $a_{m} \rightarrow \infty(m \rightarrow \infty)$, such that $(2.1)$ is right. This implies that the family $\left\{w_{m}(z):=w\left(a_{m}\right.\right.$ $+z)\}_{m \in \mathbb{N}}$ is not normal at $z=0$. By Lemma 2.1, there exist sequences $\left\{b_{m}\right\}$ and $\left\{\rho_{m}\right\}$ such that

$$
\left|a_{m}-b_{m}\right|<1, \quad \rho_{m} \rightarrow 0,
$$


and $g_{m}(\zeta):=w_{2, m}\left(b_{m}-a_{m}+\rho_{m} \zeta\right)=w_{2}\left(b_{m}+\rho_{m} \zeta\right)$ converges locally uniformly to a nonconstant entire function $g(\zeta)$, which order is at most 2 , all zeros of $g(\zeta)$ have multiplicity at least $k$. In particular, we may choose $b_{m}$ and $\rho_{m}$, such that

$$
\rho_{m} \leq \frac{2}{w_{2}^{\#}\left(b_{m}\right)}, \quad w_{2}^{\#}\left(b_{m}\right) \geq w_{2}^{\#}\left(a_{m}\right) .
$$

According to (3.6) and (3.7)-(3.9), we can get the following conclusion:

For any fixed constant $0 \leq \rho<\lambda-1$, we have

$$
\lim _{m \rightarrow \infty} b_{m}^{\rho} \rho_{m}=0
$$

In the differential equation (3.6) we now replace $z$ by $b_{m}+\rho_{m} \zeta$, then we obtain

$$
\begin{aligned}
& \left(Q\left(w_{2}^{(k)}\left(b_{m}+\rho_{m} \zeta\right)\right)\right)^{m_{1} m_{2}} \\
& \quad=\sum_{r \in I} a\left(b_{m}+\rho_{m} \zeta\right)^{m_{2}} a_{r}\left(b_{m}+\rho_{m} \zeta, g_{m}(\zeta)\right) \rho_{m}^{-p(r)} M_{r}\left[g_{m}\right](\zeta)
\end{aligned}
$$

where

$$
\begin{aligned}
Q\left(w_{2}^{(k)}\left(b_{m}+\rho_{m} \zeta\right)\right) & =\rho_{m}^{-q k}\left[\left(g_{m}^{(k)}\right)^{q}(\zeta)+\rho_{m}^{k} a_{q-1}\left(g_{m}^{(k)}\right)^{q-1}(\zeta)+\right. \\
& \left.+\cdots+\rho_{m}^{q k} a_{1} g_{m}^{(k)}(\zeta)\right] .
\end{aligned}
$$

Namely

$$
\begin{aligned}
& {\left[\left(g_{m}^{(k)}\right)^{q}(\zeta)+\rho_{m}^{k} a_{q-1}\left(g_{m}^{(k)}\right)^{q-1}(\zeta)+\cdots+\rho_{m}^{q k} a_{1} g_{m}^{(k)}(\zeta)\right]^{m_{1} m_{2}}} \\
& \quad=\sum_{r \in I} \frac{a\left(b_{m}+\rho_{m} \zeta\right)^{m_{2}} a_{r}\left(b_{m}+\rho_{m} \zeta, g_{m}(\zeta)\right)}{b_{m}^{a+d e g_{z, \infty} a_{r}}}\left\{b_{m}^{\frac{a+\operatorname{deg}_{2,2} a_{r}}{m_{1} m_{2} q k-p(r)}} \rho_{m}\right\}^{m_{1} m_{2} q k-p(r)} M_{r}\left[g_{m}\right](\zeta) .
\end{aligned}
$$

For every fixed $\zeta \in \mathbb{C}$, if $\zeta$ is not zero of $g(\zeta)$, for $m \rightarrow \infty$ and $0 \leq \rho=\frac{a+\operatorname{deg}_{z, \infty} a_{r}}{m_{1} m_{2} q k-p(r)} \leq \frac{a+\operatorname{deg}_{z_{2, \alpha} a} a}{m_{1} m_{2} q k-\operatorname{deg} P\left(w_{2}\right)}<\lambda-1$ then we have $\left(g^{(k)}\right)^{m_{1} m_{2}}=0$, which contradicts with all zeros of $g(\zeta)$ have multiplicity at least $k$. So $\lambda\left(w_{2}\right) \leq 1+\frac{a+\operatorname{deg}_{z, \infty} a}{m_{1} m_{2} q k-\operatorname{deg} P\left(w_{2}\right)}$.

The proof of Theorem 1.9 is complete.

\section{Acknowledgements}

The authors wish to thank the referees and editor for their very helpful comments and useful suggestions. This study was partially supported by Leading Academic Discipline Project (10XKJ01) and Key Development Project (12C104) of Shanghai Dianji University also was partially supported by NSFC of China (11101048 and 10771220), Doctorial Point Fund of National Education Ministry of China (200810780002). The MS ID is 6597357865695822.

\section{Author details}

${ }^{1}$ Department of Mathematics and Physics, Shanghai Dianji University, Shanghai 200240, People's Republic of China ${ }^{2}$ School of Science, Beijing University of Posts and Telecommunications, Beijing 100876, People's Republic of China ${ }^{3}$ School of Mathematics and Information Science, Guangzhou University, Guangzhou 510006, People's Republic of China

\section{Authors' contributions}

JQ carried out the main part of this manuscript. YL and WY participated discussion and corrected the main theorem. All authors read and approved the final manuscript.

\section{Competing interests}

The authors declare that they have no competing interests. 


\section{References}

1. Hayman, WK: Meromorphic Functions. Clarendon Press, Oxford (1964)

2. He, YZ, Xiao, XZ: Algebroid Functions and Ordinary Differential Equations. Science Press, Beijing (1988) (in Chinese)

3. Laine, I: Nevanlinna Theory and Complex Differential Equations. de Gruyter, Berlin (1993)

4. Gol'dberg, AA: On single-valued solutions of algebraic differential equations. Ukrain Mat Zh. 8, 254-261 (1956)

5. Bank, S, Kaufman, R: On meromorphic solutions of first-order differential equations. Comment Math Helv. 51, 289-299 (1976). doi:10.1007/BF02568158

6. Barsegian, G: Estimates of derivatives of meromorphic functions on sets of a-points. J Lond Math Soc. 34(2):534-540 (1986)

7. Barsegian, G: On a method of study of algebraic differential equations. Bull Hong Kong Math Soc. 2(1):159-164 (1998)

8. Bergweiler, W: On a theorem of Gol'dberg concerning meromorphic solutions of algebraic differential equations. Complex Var. 37, 93-96 (1998)

9. Yuan, WJ, Xiao, B, Zhang, JJ: The general result of Gol'dberg's theorem concerning the growth of meromorphic solutions of algebraic differential equations. Comput Math Appl. 58, 1788-1791 (2009). doi:10.1016/j.camwa.2009.07.092

10. Qi, JM, Li, YZ, Yuan, WJ: Further results of Gol'dberg's theorem concerning the growth of meromorphic solutions of algebraic differential equations. Acta Math Sci. (in press, in Chinese)

11. Gu, RM, Ding, JJ, Yuan, WJ: On the estimate of growth order of solutions of a class of systems of algebraic differential equations with higher orders. J Zhanjiang Normal Univ (in Chinese). 30(6):39-43 (2009)

12. Zalcman, L: A heuristic principle in complex function theory. Am Math Monthly. 82, 813-817 (1975). doi:10.2307/ 2319796

13. Pang, XC, Zalcman, L: Normal families and shared values. Bull Lond Math Soc. 32, 325-331 (2000). doi:10.1112/ S002460939900644X

14. Zalcman, L: Normal families new perspectives. Bull Am Math Soc. 35, 215-230 (1998). doi:10.1090/S0273-0979-98-00755-1

15. Gu, RM, Li, ZR, Yuan, WJ: The growth of entire solutions of some algebraic differential equations. Georgian Math J. 18(3):489-495 (2011)

doi:10.1186/1687-1847-2012-6

Cite this article as: Jianming et al:: Further results of the estimate of growth of entire solutions of some classes of algebraic differential equations. Advances in Difference Equations 2012 2012:6.

\section{Submit your manuscript to a SpringerOpen ${ }^{\circ}$ journal and benefit from:}

Convenient online submission

- Rigorous peer review

- Immediate publication on acceptance

- Open access: articles freely available online

- High visibility within the field

- Retaining the copyright to your article

Submit your next manuscript at $\mathbf{s p r i n g e r o p e n . c o m ~}$ 